\title{
ÉTICA Y POLÍTICA..., Y ALGUNOS DE SUS ALEDAÑOS
}

\author{
Alfonso Ruiz Miguel \\ Universidad Autónoma de Madrid
}

RESUMEN. El ensayo parte de los dos escritos de Garzón Valdés sobre las relaciones entre ética y política y hace dos tipos de consideraciones: primero, se analiza una referencia marginal que aparece en ellos sobre las elecciones trágicas para poner de manifiesto su difícil compatibilidad con una concepción racionalista de la moral como la defendida por Garzón Valdés; después se comparan los tipos de relación propuestos por él entre ética y política con otras afirmaciones que aparecen en su obra sobre las relaciones entre, de un lado, moral y Derecho y, de otro lado, política y Derecho.

Palabras clave: Ernesto Garzón Valdés, relaciones ética-política.

ABSTRACT. The paper takes up the two articles of Garzón Valdés about the relations between ethics and politics to propose two reflections: firstly, it analyzes some marginal references made in both articles about moral tragic choices which are hardly compatible with a rationalist conception of morals as it is asserted by Garzón Valdés; secondly, the paper compares the kinds of relationship between ethics and politics to different assertions in his work about relations between, on the one hand, morals and law and, on the other hand, politics and law.

Keywords: Ernesto Garzón Valdés, ethics-politics relations. 


\section{1. ÉTICA Y POLÍTICA}

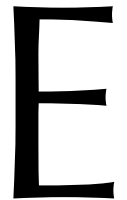

legí para mi comentario los dos escritos de Ernesto GARZÓN VALDÉS sobre ética y política, originariamente publicados entre 1984 y 1987 aunque desde que los leí me han parecido siempre plenamente clarificadores y, en realidad, inobjetables ${ }^{1}$. Pensé que sobre semejante tema siempre se pueden decir muchas cosas. Al cabo, tras mi última relectura, esos escritos por sí mismos únicamente me han suscitado una leve salvedad sobre unas esporádicas referencias a las elecciones trágicas que enseguida haré. Y así, por encima del estricto marco de ambos escritos, y a riesgo de invadir el terreno de otros comentarios, creí que podía tener mayor interés en este encuentro ojear también en los aledaños del binomio ética y política para relacionarlo con un tradicional tercero en discordia: el Derecho.

Comenzaré por recordar la esencia de los dos ensayos sobre ética y política, que afirman eficazmente la tesis del sometimiento de toda acción política a los criterios de la ética normativa (en adelante, «ética» sin más). En su esquema básico, como es característico de muchos de sus escritos, GARZÓN VALDÉS propone una combinatoria de distinciones, en este caso entre plano descriptivo y prescriptivo de un lado y entre ética y moral positiva de otro. Por destacar sólo los argumentos centrales, la tesis descriptiva de que la ética es ajena a la política sería falsa como proposición universal pero verdadera como afirmación particular referida a ciertos sistemas y comportamientos políticos; la segunda tesis descriptiva de que la moral positiva es independiente de la política sería siempre falsa porque ningún sistema político puede subsistir sin legitimación, esto es, sin la creencia en la legitimidad, o sin la adopción del hartiano punto de vista interno, al menos por parte del grupo dominante; en tercer lugar, la tesis prescriptiva de que la ética no debe, o no puede, jugar un papel relevante en la política, que constituye el núcleo y el desarrollo más extenso de los dos ensayos, es discutida por Ernesto GARZÓN respecto de las diferentes variantes de la tesis de la separación entre ética y política bajo el presupuesto conceptual, que comparto, de que los criterios morales, a diferencia de los técnicos, son los criterios racionalmente coherentes e imparciales y de carácter último para el juicio de cualquier acción, incluidas las acciones políticas; en fin, la cuarta tesis prescriptiva de que la moral social no debe jugar un papel en la política recibe la también coherente e inobjetable respuesta de que depende de si tal moral se ajusta o no a la ética.

\section{RACIONALIDAD ÉTICA Y ELECCIONES TRÁGICAS}

Como avancé al principio, tengo sólo una leve salvedad, casi apenas una duda, sobre un único punto que aparece casi incidentalmente en el desarrollo de la anterior reconstrucción. En dos momentos del primer escrito sobre «Moral y política» parece aceptarse que los criterios morales, y por tanto la supremacía de la ética sobre la política, no serían aplicables en las elecciones trágicas, es decir, en aquellas situaciones «física y lógicamente posibles» en las que cualquier curso de acción es no sólo éticamen-

\footnotetext{
1 «Moral y política», Anuario de Filosofía del Derecho, 1984, pp. 177-195; y «Acerca de la tesis de la separación entre moral y política», Sistema, n. 76, 1987; ambos ahora, por donde se citan, en Derecho, ética y política, 1993, Madrid: Centro de Estudios Constitucionales, pp. 541-560 y 561-570.
} 
te malo, ante lo que se puede elegir el mal menor, sino también injusto, esto es, lesivo para la autonomía de otras personas y, por tanto, sin salida «deónticamente posible» ${ }^{2}$. Es verdad que las elecciones trágicas son inmediatamente por GARZÓN circunscritas sólo a aquellos casos en los que existe una situación previa de injusticia. Pero aquí aparece el problema, que depende de cómo se entienda esta condición, de manera estricta o amplia.

Si la previa injusticia se debe haber cometido estrictamente por la propia persona que después se encuentra en la situación de elección trágica —el ejemplo más socorrido es la situación derivada de una promesa injusta-, no parece haber objeción alguna en admitir la posibilidad de tales elecciones trágicas. Desde que Tomás DE AQUINO las formuló como conflictos o perplejidades secundum quid, tales situaciones no parecen poner en aprietos a una concepción racionalista de la ética, que no incurre en inconsistencia por atribuir alguna responsabilidad al sujeto, sea cual sea su elección, debido a su falta previa.

En cambio, si, ampliando la condición, se trata de conflictos derivados de una injusticia histórica, estructural o, sin más, producida por terceras personas - las perplejidades simpliciter en la terminología DE AQUINO, que es el sentido habitual en el que se habla de elecciones trágicas o dilemas morales ${ }^{3}-$, me parece que la aceptación de la posibilidad de tales situaciones entra en conflicto con una concepción como la que GARZÓN VALDÉs ha mantenido en el conjunto de su obra, una concepción que comparto, basada en dos rasgos: uno, la posibilidad de una respuesta única, coherente y racional, imparcialmente universalizable, para cualquier decisión moral (bajo el criterio de que debe implica puede y salvo que uno mismo haya incurrido previamente en injusticia); y, dos, la justificabilidad última del principio de autonomía y, por tanto, de responsabilidad individual ${ }^{4}$. A mi modo de ver, si alguien no es responsable de la injusticia previa, debe poder actuar moralmente en la alternativa de dos cursos de acción incompatibles y ambos dañinos, es decir, debe ser deónticamente posible la elección del daño menor.

No tengo claro si GARZÓN VALDÉS considera la condición estricta o la amplia. Al final del segundo escrito sobre ética y política se distancia claramente de la tesis de que la acción política da lugar necesariamente a elecciones trágicas destacando que tal tesis

2 «Moral y política», p. 557.

3 En la más relevante compilación contemporánea sobre los dilemas morales, sólo en una contribución se trata el tema de los conflictos secundum quid con un mínimo detenimiento (cfr. DONOGAN, 1987: «Consistency in Rationalist Moral Systems», en Chistopher W. Gowans [comp.], Moral Dilemmas, Nueva York, etc., Oxford University Press, pp. 284-185, donde menciona An Essay in Deontic Logic..., de VON WRIGHT como una de las primeras, escasas y relevantes referencias contemporáneas al criterio de AQUINO; por cierto que es a ese mismo ensayo al que remite GARZÓN cuando menciona por primera vez las elecciones trágicas en «Moral y política», cit., p. 557, nota 35). En aquella compilación, salvo esporádicas y muy limitadas alusiones (cfr. «Preface», ibidem, p. vii; BARCAN MARCuS: «Moral Dilemmas and Consistency», ibidem, p. 193; HaRE: «Moral Conflicts», ibidem, p. 207), los dilemas morales corresponden siempre a los conflictos simpliciter, que ocurren porque dos obligaciones o deberes morales no son realizables a la vez dadas las circunstancias de hecho y sin previa falta por parte del sujeto.

${ }^{4}$ En la elaborada reconstrucción de ATIENZA: «La filosofía moral de Ernesto Garzón Valdés» (Introducción a Derecho, ética y política, cit., pp. 21-32), ambas afirmaciones corresponden a grandes rasgos a los principios $\mathrm{P}_{7}$ (y, en especial, de las tesis derivadas $\mathrm{T}_{20}$ a $\mathrm{T}_{22}$ ), $\mathrm{P}_{8}$ (y, en especial, de la regla $\mathrm{R}_{23}$ y de las tesis $\mathrm{T}_{23}$ y $\mathrm{T}_{24}$ ), $\mathrm{P}_{9}$ (y, en especial, las tesis $\mathrm{T}_{25}$ y $\mathrm{T}_{26}$ ) y $\mathrm{P}_{10}$ ( $\mathrm{y}$, en especial, la tesis $\mathrm{T}_{28}$ ). 
«conduce a la sorprendente conclusión» de que la moral no puede resolver racionalmente los conflictos y comete «el error de atribuir a las normas éticas carácter incondicionado» ${ }^{5}$. Suscribo el argumento. Sin embargo, también al final del primer escrito parece asumir la condición amplia cuando afirma que las armas modernas hacen imposible la distinción entre combatientes y no combatientes y que por tanto las guerras actuales conducen fatalmente a elecciones trágicas ${ }^{6}$. Pero las guerras son, típicamente, situaciones de cuya iniciación sólo unos pocos son responsables, de modo que mucha gente puede verse después activamente envuelto en ellas sin previa responsabilidad personal. ¿También estos participantes posteriores, por llamarlos así, se colocan ante posibles elecciones trágicas?

La consecuencia de ese argumento no sería sólo, como concluye GARZÓN VALDÉS y luego ha desarrollado en Calamidades $^{7}$, que hoy todas las guerras son inmorales, de modo que sus responsables actuarían injustamente. Aunque esto me parece discutible respecto de los dirigentes que ordenan la defensa frente a una agresión, lo dejo a un lado aquí para detenerme sólo en el caso de los participantes en esa defensa. Porque de tal argumento parece deducirse también que ninguna persona podría participar en una guerra, aun no siendo responsable de su provocación, so pena de incurrir por ello en una injusticia que le coloca ante posibles elecciones trágicas. Sin embargo, ante una agresión injusta, a muchas personas, incluido yo mismo, aceptar ese criterio las situaría ante una elección previa no provocada por ellas y, aunque quizá trágica en un sentido trivial, moralmente resoluble: cómo elegir entre intentar evitar la injusticia de la agresión (supóngase, una invasión como la nazi, que afecta a la vida y la libertad de los conciudadanos) y evitar la injusticia de tomar las armas frente a la agresión. A mi modo de ver, si, con GARZÓn VALDÉs, aceptamos el criterio de la unidad y la racionalidad de la moral, estas situaciones son deónticamente resolubles y lo son con los principios de responsabilidad individual y de la licitud de repeler la violencia con la violencia. De lo contrario, me parece, las elecciones trágicas serían posibles sin responsabilidad individual previa y el razonamiento moral no sería reconducible a una unidad racional.

\section{DE LA ÉTICA Y LA POLÍTICA AL DERECHO}

Comentada la salvedad relativa a un punto en realidad menor de los dos artículos sobre ética y política, me sitúo ahora en un plano mucho más general para conectar las tesis mantenidas en aquellos textos con el tema del Derecho. Si Derecho, ética y política es, precisamente, el título de la gran compilación de textos de GARZÓN VALDÉs publicada en 1993, así como de este seminario, el binomio ética y política abre una doble pregunta respecto de los otros dos binomios posibles de la triada: primera, con el binomio ética y Derecho, sobre el que existen otros dos importantes escritos de Ernesto GARZÓN, y, segunda, con el binomio política y Derecho, del que no aparece explícitamente ningún escrito en su bibliografía. Veámoslo por partes.

\footnotetext{
5 «Acerca de la tesis de la separación...», cit., p. 570.

${ }^{6}$ Cfr. «Moral y política», cit., p. 560.

7 Barcelona: Gedisa, 2004.
} 


\subsection{Derecho y ética}

Es casi innecesario decir que las relaciones entre Derecho y ética son un semillero, si no también un avispero, de polémicas y problemas enmarañados y aparentemente inacabables. GARZÓN VALDÉs ha dedicado específicamente al problema dos ensayos, publicados en 1993 y en 1996, de los que el segundo, bastante más amplio, integra las tesis del primero ${ }^{8}$. Me voy a fijar únicamente en las tesis del primer escrito, muy relacionadas entre sí, porque conectarlas con las aportaciones de los escritos sobre ética y política que antes he sintetizado pone de relieve algunas diferencias respecto de la relación entre Derecho y ética que merecen comentario. Me refiero a la doble tesis mantenida en él de que tanto la necesaria asunción del punto de vista interno como la pretensión de corrección de las normas jurídicas, en ambos casos al menos para los gobernantes del sistema, implicarían una necesaria vinculación conceptual entre Derecho y ética 9 .

Concuerdo en que tanto el punto de vista interno como la pretensión de corrección son genuinamente morales para quienes los suscriben, en la medida en que, en efecto, no los conciben como razones prudenciales o como meras apariencias engañosas. Pero llama la atención la diferencia en las dos clases de conexión destacadas en los escritos sobre ética y política, la empírica y la normativa, y una tercera forma de conexión, la conceptual, que es la única que aparece como relevante en la relación entre Derecho y ética. Más allá de la razón de esa diferencia, atribuible al enfoque tradicional en la polémica filosófico-jurídica entre positivismo y iusnaturalismo, contrastar ese planteamiento con el de la relación entre ética y política, y en especial la aproximación empírica ante esa relación con la conceptual ante la relación entre Derecho y moral, quizá pueda aclarar un poco esta ya de por sí intrincada cuestión en lo que concierne a dos puntos.

Ante todo, lo primero que salta a la vista en el paso de una a otra relación es que el cambio de la perspectiva empírica a la conceptual —es decir, de una perspectiva meramente descriptiva a una meramente definitoria - no puede comportar transformación alguna respecto del tipo de moral del que se está hablando: a mi modo de ver, en los dos casos de la moral positiva (al menos de los dirigentes) del sistema político o jurídico en cuestión, y no de la moral crítica, que no puede ser otra que la ética desde el punto de vista del teórico que afirma la conexión. Mientras la tesis descriptiva afirma que una cierta moral positiva es necesaria para la existencia de un régimen político, la tesis conceptual mantiene que no puede llamarse jurídico a un régimen que carezca de pretensión de corrección y en el que sus sostenedores no adopten el punto de vista interno. Pero si quien hace tales afirmaciones no adopta tal criterio moral como propio, de modo que no asume la tesis fuerte según la cual no puede existir un sistema político o jurídico inmoral (desde su propio punto de vista), lo único que está destacando, sea

${ }^{8}$ «Weitere Überlegungen zur Beziehung zwischen Recht und Moral», en AARNIO, A., et alia (comps.), 1993: Rechtsnorm und Rechtswirklichkeit. Festschrift für Werner Krawietz zum 60. Geburtstag, Berlín: Duncker \& Humblot; ahora, por donde se cita, como «Algo más acerca de la relación entre Derecho y moral», en Derecho, ética y política..., cit., pp. 317-334; y «Derecho y moral», en GARZÓN VALDÉS y LAPORTA (comps.), 1996: El Derecho y la justicia, Madrid: Trotta, pp. 397-424.

${ }^{9} \mathrm{Cf}$. «Derecho y moral», cit., que comienza caracterizando expresamente la polémica no como empírica sino como «relativa a la posibilidad o imposibilidad de establecer una relación conceptual entre Derecho y moral» (p. 397; cfr. también pp. 407-409; así como «Algo más acerca de la relación...», cit., p. 324). 
descriptiva o sea conceptualmente, es la importancia de la legitimación en el sentido weberiano de la palabra, esto es, de la creencia en la legitimidad de tales sistemas por parte de al menos algunos de los asociados. Y la creencia en la legitimidad no puede ser asumida sin más por parte del teórico como legitimidad genuina so pena de deponer todo criterio moral propio en favor de cualquier moral positiva ${ }^{10}$.

El segundo punto que merece considerarse es el de la propia relación entre la conexión empírica y la conceptual. ¿No es la segunda parasitaria de la primera, al menos en el sentido de que si la conexión empírica se considera necesaria, la conexión conceptual se deriva necesariamente de ella? Así lo creo. Pero obsérvese que no estoy afirmando la total identidad entre la perspectiva empírica y la conceptual, sino una conexión unidireccional. En efecto, aquella derivación no parece operar en la dirección inversa, esto es, en el sentido de que si no se considera necesaria la conexión empírica es perfectamente posible mantener por estipulación una conexión conceptual entre Derecho y moral (y tanto de la moral positiva del sistema como de la moral crítica desde el punto de vista del teórico). Pero si se mantiene la necesidad de una determinada conexión empírica no sería razonable definir el Derecho (o la política) excluyendo esa condición del concepto ${ }^{11}$. Conforme a ello, lo que GARZÓN VALDÉs ha hecho es adoptar como uno de los criterios definitorios del Derecho el rasgo empírico de la adopción de un criterio moral por parte de los sostenedores del sistema jurídico.

\subsection{Derecho y política}

Me resta concluir con el tercer binomio, precisamente entre Derecho y política, al que, si no me equivoco, GARZÓN VALDÉs no ha dedicado ningún escrito específico, aunque sí puedan rastrearse alusiones sustantivas a él en distintos lugares. Antes de verlo, enmarco mi comentario. Entre las distintas formas generales de concebir la relación entre política y Derecho dos me parecen especialmente relevantes. Para la que llamaría tesis de la correspondencia, mantenida por ejemplo por BoBBIo bajo la imagen de la cara y la cruz de la misma moneda, poder político y Derecho estarían en un mismo nivel, en una relación de interdependencia y soporte mutuo que, además, los diferencia-

\footnotetext{
${ }_{10}$ Aunque esta diferencia entre legitimación y legitimidad es la sustancia de la tesis expresamente asumida por GARZÓN VALDÉS en sus escritos sobre Derecho y moral, hay una cierta insistencia en la conexión conceptual entre Derecho y ética (y no meramente entre Derecho y moral positiva) que la oscurecen innecesariamente: así, por dar un ejemplo bastante claro, cuando dice que la tesis de la pretensión de corrección moral como condición necesaria de la existencia de cualquier sistema jurídico refuta la negación de que tanto la moral positiva como la ética jueguen algún papel en el concepto de Derecho ( $c f r$. «Algo más acerca de la relación», cit., pp. 329-330), una idea que sólo puede aceptarse si se refiere a la ética desde el punto de vista de autoridades y funcionarios, es decir, a una moral social, que por tanto no tiene por qué ser un criterio legítimo desde el punto de vista de un observador (en este sentido, en cambio, $c f r$. «Derecho y moral», cit., pp. 408-409).

${ }^{11}$ Paralelamente, quien acepte el argumento empírico de Ralf SARTORIUS de que para la subsistencia de un sistema jurídico no es necesaria la asunción genuina del punto de vista interno, porque bastaría con que todos los participantes hicieran como si lo asumieran, aunque bajo la creencia conjunta de que los demás sí lo asumen genuinamente ( $c f r$. «Positivism and the Foundations of Legal Authority», en GAVISON [comp.] 1987, Issues in Contemporary Legal Philosophy. The Influence of H. L. A. Hart, Oxford: Clarendon Press, pp. 51-52), también debe aceptar la conexión conceptual entre el Derecho y la no negación expresa del punto de vista interno por parte de los operadores del sistema (así lo hice yo mismo en «Derecho y punto de vista moral», Anuario de Filosofía del Derecho, tomos XIII-XIV, 1996-1997, p. 578).
} 
ría a ambos de la ética, con la que no se podrían identificar, si bien la ética sería la piedra de toque última de uno y otro ${ }^{12}$. Por su parte, para la tesis que llamaría de la superación, mantenida por ejemplo por PASSERIN D'ENTRÈVES, mientras la política se caracterizaría en último término maquiavelianamente, por el recurso a la fuerza y el engaño, en cambio, el Derecho ocuparía un lugar intermedio entre la política y la ética, añadiendo a la política la exigencia del gobierno de las leyes y, sin ser sólo por ello completamente legítimo, compartiendo al menos algunos requisitos básicos de la ética a través de unos mínimos de seguridad y orden ${ }^{13}$.

¿Cuál es la posición adoptada por GARZÓn VALDÉS ante esa alternativa? Creo que puede detectarse una cierta ambigüedad, incluso en escritos cercanos en el tiempo. Por una parte, hay una línea bastante persistente que podría incluirse en la primera opción, según la cual tanto los regímenes políticos como los jurídicos están sometidos a condiciones similares de existencia y legitimación pero no de legitimidad: su existencia exige legitimación pero no, en absoluto, legitimidad, que puede o no darse según su adecuación con la ética ${ }^{14}$.

Pero, por otra parte, en algunos de sus textos hay otra línea que parecería seguir más bien la segunda opción sobre la relación entre política y Derecho, atribuyéndole a este último unos mínimos rasgos de legitimidad que no se presuponen para todo sistema político. En efecto, si por un lado ha afirmado que «no son pocos los sistemas políticos pasados o presentes que carecen de legitimidad. Los ejemplos de Nerón, Hitler, Idi Amín, del apartheid o de Rafael Videla pueden multiplicarse fácilmente» ${ }^{15}$, por otro lado ha aceptado el argumento de que todo Derecho, incluso las leyes raciales de Núremberg, garantiza una básica seguridad jurídica y por tanto tiene una conexión conceptual con una moral mínima ${ }^{16}$. Tal vez una y otra posición pueden ser coherentes entre sí a través de la tesis, también defendida por GARZÓN en varios lugares, de que sólo el terrorismo de Estado nos retrotrae a la situación prejurídica del estado de naturaleza hobbesiano, es decir, a una negación radical del Derecho ${ }^{17}$.

12 Véase Bоввіо, 1964: «Sul principio di legitimità», en Studi per una teoria generale del diritto, Turín: Giappichelli, G.: 1970, $\$$ 5, trad. cast. en Contribución a la teoría del Derecho, 1990, ed. de RuIZ Miguel, A. 2. ${ }^{a}$ ed., Madrid: Debate, 1990; 3. ${ }^{a}$ ed, Puebla (México): Cajica, 2006; véase también «Dal potere al diritto e viceversa» (1981); en Teoria generale della politica, 1999, ed. de Bovero, Turín: Einaudi, IV.ii, trad. cast., Teoría general de la política, Madrid: Trotta, 2003; también en Contribución... cit., cap. XVII. Sobre ello puede verse también RuIZ Miguel, 2005: «Del Derecho al poder: el camino central de la obra de Norberto BoBBIO», Doxa. Cuadernos de Filosofía del Derecho, n. 28, 2005, pp. 59-71.

${ }_{13}$ Véase PASSERIN D'ENTRÈVES, 1967: The Notion of the State. An Introduction to Political Theory, Oxford: Oxford University Press, 1967; en versión italiana, La dottrina dello stato. Elementi di analisi e di interpretazione, 2. ${ }^{a}$ ed., Turín: Giappichelli, 1967, trad. cast. de la ed. inglesa y prólogo de PUNSET, 2001: La noción de Estado. Una introducción a la Teoría Política, Barcelona: Ariel, 2001.

${ }^{14}$ La tesis aparece en paralelo en los escritos sobre ética y política ( $c f r$. «Algo más acerca de la relación...» cit., pp. 328-329) y sobre moral y Derecho (cfr. «Acerca de la tesis de la separación...», cit., p. 563), así como, conectando de manera más expresa sistema político y jurídico, en «Hermann Heller y John Austin: un intento de comparación» (1983) y en «El concepto de estabilidad de los sistemas políticos» (1987), ambos ahora, por donde se citan, en Derecho, ética y política, cit., pp. 163 y 178; y $573-577$ y $580-588$, respectivamente. Una frase de este último escrito resume bien la tesis a que aludo en el texto: «la adopción del punto de vista interno y su capacidad de imposición en la sociedad son condiciones necesarias y suficientes para la existencia del sistema. No se dice nada acerca de su estabilidad y tampoco acerca de su legitimidad» (pp. 587-588).

15 «Acerca de la tesis de la separación...», cit., p. 562; la cursiva es mía.

${ }^{16}$ Cfr. «Derecho y moral», cit., pp. 402-403.

${ }^{17}$ Cfr. ibidem, pp. 403 y $409-410$. 
Esta segunda línea plantea una cuestión muy compleja que habría que meditar más pausadamente. Como no estoy seguro de que cuadren todas las cuentas me limitaré a dejar anotadas algunas de mis dudas: caquella situación prejurídica no es también prepolítica?, ¿la pretendida seguridad mínima del Derecho es un rasgo empírico necesario o un elemento ético-normativo de su concepto?, ¿se puede reducir la ilegitimidad de un régimen político al terrorismo del Estado?, ¿`i en la ilegitimidad hay grados, no los hay también en el terrorismo de Estado?, ¿es lo mismo terrorismo de Estado que Estado terrorista?, ¿en un sistema globalmente ilegítimo, no son una cosa las leyes en el papel y otra las prácticas, y no son más relevantes para su caracterización éstas que aquéllas?, ¿el régimen nazi en particular, no pasó desde la mínima seguridad jurídica de las leyes racistas de septiembre de 1935, que negaban la ciudadanía a los judíos y prohibían las relaciones matrimoniales y sexuales entre los judíos y los «ciudadanos de sangre alemana y afín», hasta algo igual o peor que un Estado terrorista cuando comenzó la deportación y el exterminio por razones raciales y políticas? En fin, nadie mejor que el propio Ernesto GARZÓN VALDÉS para responder a estas y otras preguntas similares, a las que añadiría esta última, de naturaleza bien diferente: ¿por qué no en un artículo específicamente dedicado a la relación entre política y Derecho? 